Article

\title{
Comprehensive Performance Quasi-Non-Volatile Memory Compatible with Large-Scale Preparation by Chemical Vapor Deposition
}

\author{
Kun Yang, Hongxia Liu *(D), Shulong Wang * , Wenlong Yu and Tao Han \\ Key Laboratory for Wide-Band Gap Semiconductor Materials and Devices of Education, \\ The School of Microelectronics, Xidian University, Xi'an 710071, China; kuny2019@163.com (K.Y.); \\ 13772406590@163.com (W.Y.); taohan373@gmail.com (T.H.) \\ * Correspondence: hxliu@mail.xidian.edu.cn (H.L.); slwang@xidian.edu.cn (S.W.); \\ Tel.: +86-130-8756-8718 (H.L.); +86-150-9115-4611 (S.W.)
}

Received: 6 July 2020; Accepted: 24 July 2020; Published: 27 July 2020

\begin{abstract}
Two-dimensional materials with atomic thickness have become candidates for wearable electronic devices in the future. Graphene and transition metal sulfides have received extensive attention in logic computing and sensing applications due to their lower power dissipation, so that their processes have been relatively mature for large-scale preparation. However, there are a few applications of two-dimensional materials in storage, which is not in line with the development trend of integration of storage and computing. Here, a charge storage quasi-non-volatile memory with a lanthanum incorporation high-k dielectric for next-generation memory devices is proposed. Thanks to the excellent electron capture capability of $\mathrm{LaAlO}_{3}$, the $\mathrm{MoS}_{2}$ memory exhibits a very comprehensive information storage capability, including robust endurance and ultra-fast write speed of $1 \mathrm{~ms}$ approximately. It is worth mentioning that it exhibits a long-term stable charge storage capacity (refresh time is about $1000 \mathrm{~s}$ ), which is $10^{5}$ times that of the dynamic random access memory (refresh time is on a milliseconds timescale) so that the unnecessary power dissipation greatly reduces caused by frequent refresh. In addition, its simple manufacturing process makes it compatible with various current two-dimensional electronic devices, which will greatly promote the integration of two-dimensional electronic computing.
\end{abstract}

Keywords: $\mathrm{MoS}_{2}$; non-volatile memory; charge trapped; Raman spectrum; PL spectrum; chemical vapor deposition; hysteresis effect

\section{Introduction}

As the size of devices continues to decrease, the difficulty of traditional silicon-based processes continues to increase and is close to its physical limit. There is an urgent need to explore a new channel material to replace silicon, so that Moore's Law can be continued [1]. At present, the small-size silicon-based transistor uses the Fin-FET structure, which can improve the control ability of the gate to the channel, and greatly improve the performance of the device [2]. Based on this idea, a wide range of researchers have began to seek a suitable two-dimensional semiconductor to effectively improve the integration of the chip [3,4]. Since the discovery of graphene, a large number of layered crystals have been stripped in order to make up for the shortcomings of graphene band gap, therefore the $\mathrm{MoS}_{2}$ is a suitable candidate. The energy band structure of $\mathrm{MoS}_{2}$ depends on the number of layers and the single-layer $\mathrm{MoS}_{2}$ has a direct band gap, which undoubtedly provides huge opportunities for electrical and optoelectronics [5]. In recent years, a lot of research has been carried out surrounding $\mathrm{MoS}_{2}$, and various new electronic devices based on $\mathrm{MoS}_{2}$ have emerged, including 
gas sensors, resistive memory, photodetectors, etc. [6-12]. A lot of research is currently focused on how to make $\mathrm{MoS}_{2}$ transistors provide high-performance logic computing power and try to improve the performance of transistors through various process methods, which also brings a series of achievement simultaneously [13-16]. However, there are few studies on the application of $\mathrm{MoS}_{2}$ in storage, and only limited research on $\mathrm{MoS}_{2}$ non-volatile memory has been proposed [17-19].

With the rapid development of the information age, the huge amount of data presents new challenges to memory technology, especially for storage speed and data retention ability. For volatile memory, the lower integration and high cost have limited its application in cache, whereas the high power consumption obscures the advantages of DRAM due to poor data retention time [20,21]. Therefore, there is an urgent need to propose a comprehensive memory to fill the time-scale gap between volatile and non-volatile memory. So far, the memory based 2D materials were mostly based on the float gate structure with a slow write speed. Simone Bertolazzi et al. proposed a floating gate transistor using graphene as a trap layer with stable information storage capability, but its writing speed is only $100 \mathrm{~ms}$, far lower than the current commercial flush write speed (about $100 \mu \mathrm{s}$ ). Chunsen Liu et al. proposed a kind of semi-floating gate memory with ultra-fast write speed by an embedded $\mathrm{p}-\mathrm{n}$ junction, but the leakage current of p-n junction limits the date retention time to only $14 \mathrm{~s}[22,23]$. Additionally, the complicated preparation process is based on mechanical peeling making it difficult to prepare on a large scale, which cannot be accepted for commercialization.

In this work, a novel memory device based on the monolayer $\mathrm{MoS}_{2}$ was proposed utilizing a lanthanum incorporation high-k dielectric to provide capture traps [24,25]. The memory cells exhibit an impressive performance with large memory windows of more than $3 \mathrm{v}$ (operating voltage from -6 to $6 \mathrm{v}$ and back to $-6 \mathrm{v}$ ) and high program/erase radio of approximately $10^{3}$ applied $1 \mathrm{~ms}$ voltage pulse. During repeated program/erase operations, no performance degradation is observed and it has great data retention capabilities, indicating that the memory cell is very robust. The proposed device promotes the integration of two-dimensional integrated circuits development trend.

\section{Experiment Method}

\subsection{The Growth of $\mathrm{MoS}_{2}$ by APCVD and Materials Characterization}

The growth of $\mathrm{MoS}_{2}$ uses the traditional atmospheric pressure chemical vapor deposition (APCVD) method by a dual-temperature zone tube furnace, as shown in Figure 1a. The sulfur powder, molybdenum trioxide $\left(\mathrm{MoO}_{3}\right)$ is used as the sulfur source and molybdenum source, respectively. Two quartz boats containing $0.1 \mathrm{~g}$ sulfur powder and $2 \mathrm{mg} \mathrm{MoO}_{3}$ were put into the tube furnace, where the quartz boat with sulfur powder was located in the low temperature area, and the other quartz boat carrying $\mathrm{MoO}_{3}$ and the cleaned substrate was located in the high temperature area. During the growth process, the temperature in the low temperature zone and the high temperature zone are set to 200 and $720^{\circ} \mathrm{C}$, respectively, to ensure that the growth source sublimates. The detailed temperature control of the growth process that can be seen in Figure 1b,c shows the SEM image of the triangle monolayer $\mathrm{MoS}_{2}$ by APCVD. The Raman spectroscopy is shown in Figure 1d, exhibiting that the wavenumber difference of two peak positions is $17.9 \mathrm{~cm}^{-1}$. Figure $1 \mathrm{e}$ shows that the PL intensity at a different energy, from which the peak intensity is located at $1.84 \mathrm{eV}$, is the typical bandgap of the monolayer $\mathrm{MoS}_{2}$ [26].

\subsection{Device Fabrication and Electronic Characterization}

The silicon substrate covered with $\mathrm{LaAlO}_{3}$ is used as a new substrate for device fabrication, where $\mathrm{La}\left({ }^{\mathrm{i}-} \mathrm{PrCp}\right)^{3}, \mathrm{TMA}$ as the $\mathrm{Al}$ precursor and La precursor, respectively while $\mathrm{O}_{3}$ was used as the oxidant. Prior to the deposition of $\mathrm{LaAlO}_{3}$ dielectric, native $\mathrm{SiO}_{2}$ on the substrate was removed and immersed in a diluted HF solution ( $\mathrm{HF}: \mathrm{H}_{2} \mathrm{O}=1: 50$ ) for $50 \mathrm{~s}$ and the $\mathrm{HF}$ solution was rinsed out with de-ionized water. Post-deposition rapid thermal annealing was carried under $\mathrm{Ar}_{2}$ atmosphere at $800{ }^{\circ} \mathrm{C}$ for $60 \mathrm{~s}$. Then, the thickness of $\mathrm{LaAlO}_{3}$ was approximately $48 \mathrm{~nm}$ measured by the spectroscopic ellipsometry 
(SE) system. The characteristic of the $\mathrm{LaAlO}_{3}$ film was displayed in Figures S1-S4 (in Supplementary Materials). After the high-k dielectric deposition and material synthesis, the source-drain electrode was patterned by electron-beam lithography, followed by Ti $(10 \mathrm{~nm}) / \mathrm{Au}(50 \mathrm{~nm})$ deposited by electron-beam evaporation. The electronic characteristic of memory cells was measured by the Agilent B1500A analyzer (Santa Clara, CA, USA) at room temperature.

(a)

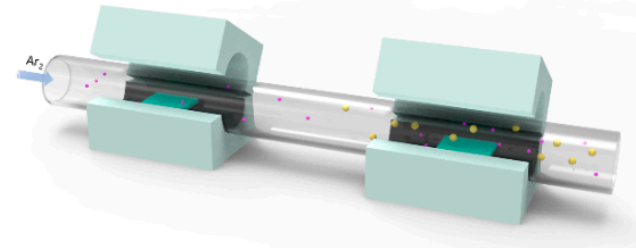

(c)

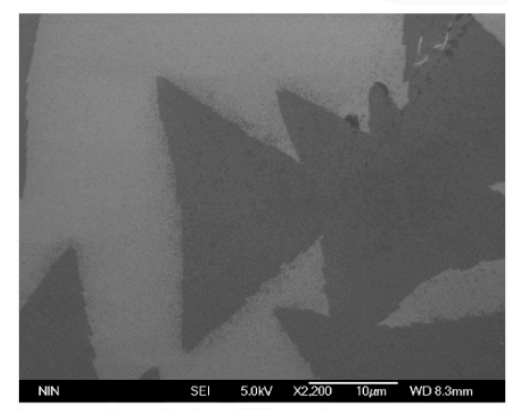

(d)

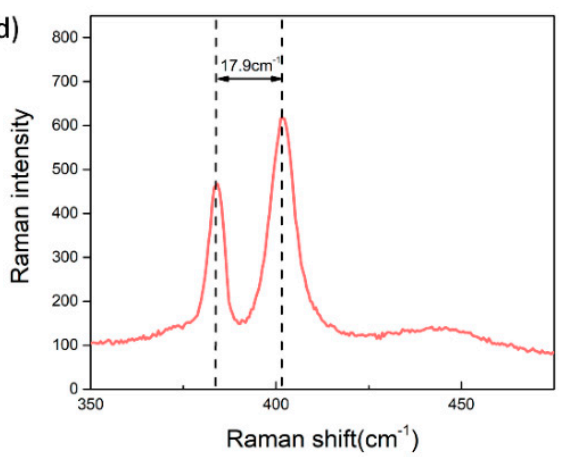

(b)
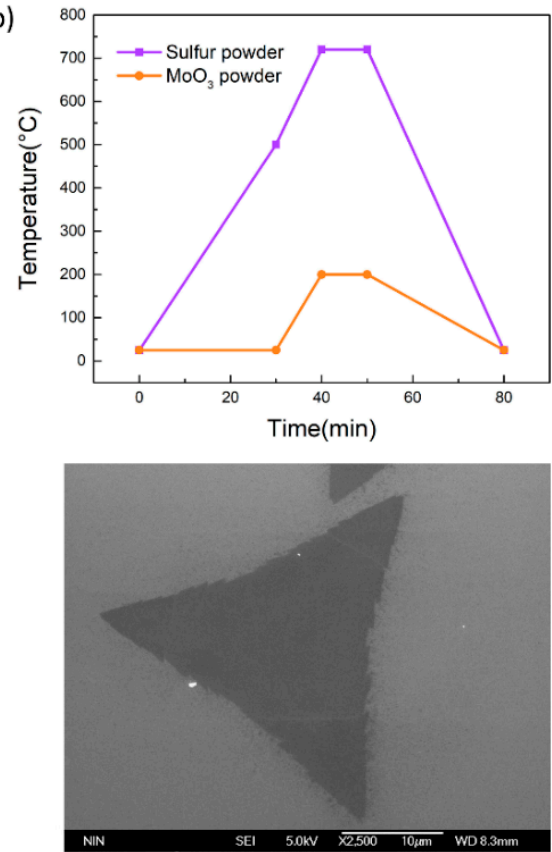

(e)

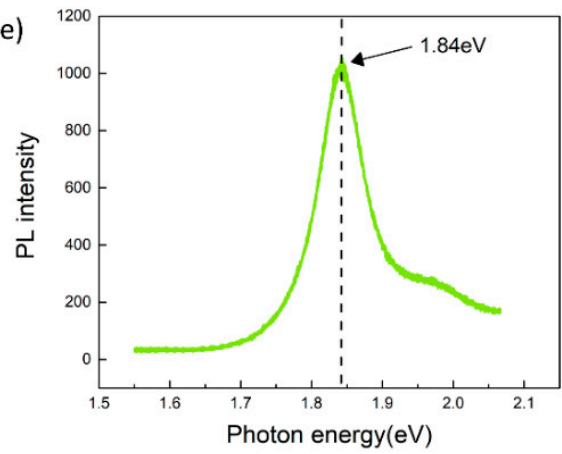

Figure 1. (a) The schematic diagram of $\mathrm{MoS}_{2}$ synthesis by atmospheric pressure chemical vapor deposition (APCVD). (b) Detailed temperature control of the growth process. (c) A highly uniform monolayer $\mathrm{MoS}_{2}$ in the scanning electron microscope (SEM). (d) The Raman spectrum of the monolayer $\mathrm{MoS}_{2}$ of two peak positions is $17.9 \mathrm{~cm}^{-1}$. (e) The PL spectrum of the monolayer $\mathrm{MoS}_{2}$ with a $1.84 \mathrm{eV}$ bandgap.

\section{Results and Discussion}

Figure 2a shows the diagram of the $\mathrm{MoS}_{2} / \mathrm{LaAlO}_{3} / \mathrm{P}^{+}-\mathrm{Si}$ memory cells. In order to analyze the electric characteristic of the device, a double voltage sweep measure was performed. Figure $2 b$ shows the SEM image of the memory cell. The information storage ability of the memory device can be obtained from transfer characteristics at different voltage peaks. As shown in Figure $2 c$, the on-off ratio up to $1 \times 10^{4}$ can be obtained when the gate voltage was swept from -6 to $6 \mathrm{v}$. For a better display effect, the $X$-axis coordinates start from $-3 \mathrm{v}$. Even if the no gate voltage is applied, the ratio of forward current and negative current exceeds $1 \times 10^{3}$, enabling the performance of 'reading-operation' only applied by the drain voltage, which is essential for reducing power consumption in the integrated 
circuit. It can be seen from Figure $2 \mathrm{c}$ that the threshold voltage drifted to some extent and the threshold voltage drifted more severely with the increase of sweep voltage peaks. When the gate voltage was swept from negative to positive, a large negative voltage was applied to the gate electrode, causing the separation of an electron-hole pair. Due to the smaller effective mass and higher mobility, the electrons transfer to the channel by tunneling under the influence of a strong electric field. On the contrary, a positive high-field makes the electrons tunnel to gate-dielectric. Therefore, with different peak voltages applied, the device threshold voltage will drift at different degrees, determined by the number of electrons tunneling from channel to oxide [27-29]. Another typical characteristic of the threshold voltage drift is that the threshold voltage drift is very small when the voltage was swept from negative to positive (hole windows shown in Figure 2c), but when the voltage was swept from positive to negative, the threshold voltage drift is large (electron windows shown in Figure 2c). This is because the tunneling process is mainly led by the electrons, and the holes are not easy to tunnel due to their large effective mass. The relationship between memory windows and sweep voltage is illustrated in Figure 2d, from which we can find obviously that the threshold voltage drifts slightly caused by hole tunneling. The memory window is determined dominantly by electron tunneling, increasing linearly with the rising of the gate voltage. When the gate voltage was swept from -6 to $6 \mathrm{v}$ and back to $-6 \mathrm{v}$, a large memory windows up to $3.3 \mathrm{v}$ is obtained. The large $\mathrm{V}_{\text {th }}$ shift windows is indispensable for a robust memory device. So as to comprehensively evaluate the storage performance memory cells, we conducted a dynamic storage test. According to the transfer characteristic of the device, by sweeping the voltage from -6 to $6 \mathrm{v}$ and back to $-6 \mathrm{v}$, not only a switch ratio of approximate 1000 can be obtained, but a large memory windows exceeding $3 \mathrm{v}$ ensures the stability of the programming operation. Hence, in the dynamic storage performance test, we chose $+6 \mathrm{v},-6 \mathrm{v}$ as the program voltage and erase voltage, respectively. Since a superior switching ratio has been obtained, which allows us still to fix $V_{D}=100 \mathrm{mV}$ as the read voltage. Figure 3a shows the input signal for the erase operation in one cycle, where a rectangular pulse with a peak voltage of $6 \mathrm{v}$ and a pulse width of $1 \mathrm{~ms}$ is used for the erase operation. After that millisecond, a rectangular pulse with a peak voltage of $0.1 \mathrm{v}$ and a pulse width of $1 \mathrm{~ms}$ is applied to the drain for the read operation. Figure $3 \mathrm{c}$ shows the output signal when the device is in the 0 -state, from which an output current below $1 \times 10^{-11}$ is obtained for 3-4 ms, but at other times, the current noise signal is detected because of the no read voltage applied. Similarly, a pulse with a peak voltage of $-6 \mathrm{v}$ and a pulse width of $1 \mathrm{~ms}$ is used for the storage operation, as shown in Figure $3 \mathrm{~b}$. When performing the read operation, an output signal of up to $1 \times 10^{-8}$ is obtained for information storage, as shown in Figure 3d. At the same time, we can find that the intensity of the output signal always maintains a good consistency during applying the read voltage with a pulse width of $1 \mathrm{~ms}$, which is vital for information storage. Otherwise, if the output signal is unstable during a read operation, it may cause data loss, especially for the 1-state.

Endurance testing is essential for a high-performance non-volatile memory, which is closely related to the number of programming. Since the programming is mainly dominated by electron tunneling, it is inevitable that the gate oxide layer will be damaged during the process of electron tunneling. For this consideration, we conducted a $\mathrm{MoS}_{2}$ memory cell endurance test. In order to speed up the progress of the test during the test, we use the B1510A module to generate a voltage pulse with a width of $1 \mathrm{~ms}$ for program and erase and the waiting time is still maintained for $1 \mathrm{~ms}$ to guarantee the data retention capability of the memory cell. The reading operation is completed by the I-V-list measurement mode, and only one sampling is performed for the given reading voltage after the waiting time is completed. Figure $3 e$ shows the trend of 1-state current and 0-state current with the number of programming times in a thousand cycles, from which the 1-state current is stability and not any performance reduction is observed. Although the 0 -state current produces small fluctuations, it is always lower than $1 \times 10^{-11}$, which guarantees a clear $0-1$ state. 
(a)

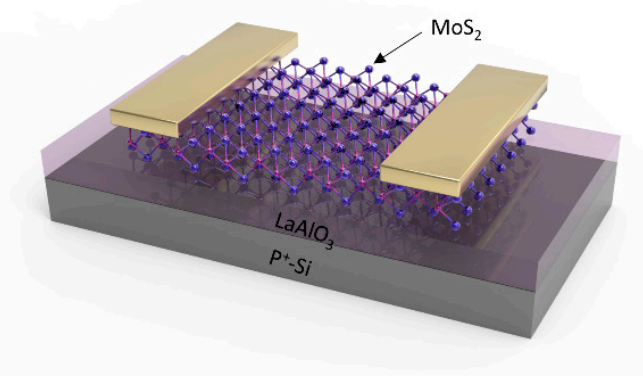

(c)

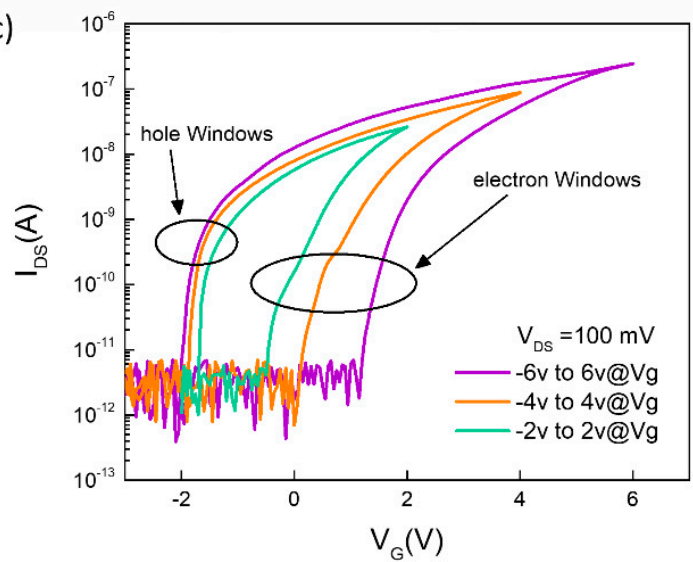

(b)

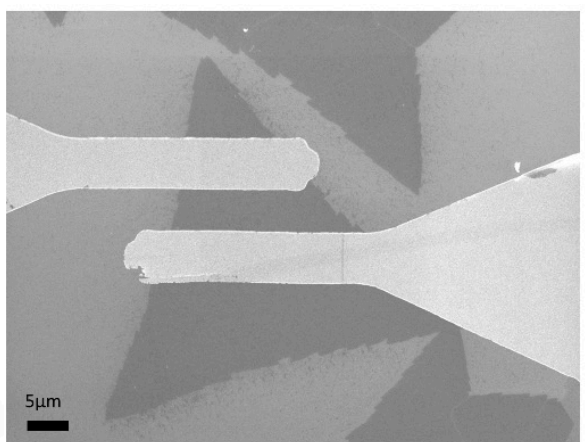

(d)

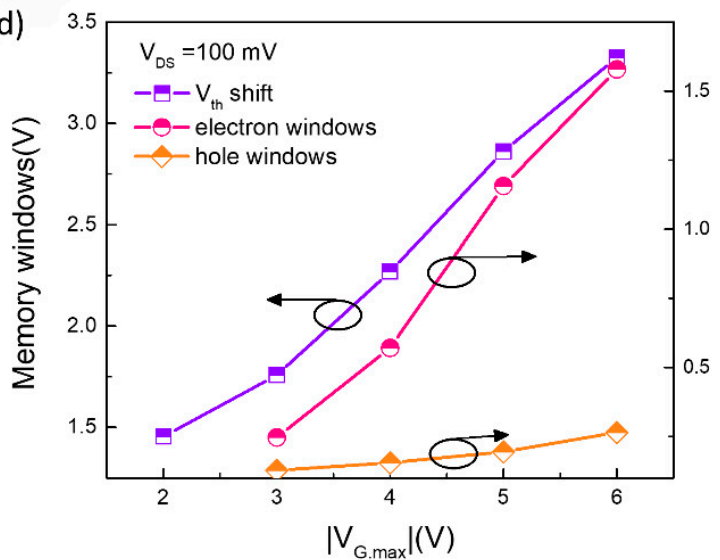

Figure 2. (a) The schematic diagram of memory cell with the $\mathrm{MoS}_{2}$ channel and $\mathrm{LaAlO}_{3}$ charge trapped layer. (b) The memory cell in the scanning electron microscope (SEM). (c) The double direction transfer characteristic at different sweep voltages. (d) The memory windows as a function of the voltage $\left|\mathrm{V}_{\mathrm{G} . \mathrm{MAX}}\right|$. The initial threshold corresponding to the threshold voltage sweeping the gate voltage \pm 2 .

Data retention capability is an important indicator for evaluating the performance of non-volatile memory. The capability of data retention is directly related to the frequency at which the memory needs to be powered on and written, which will directly determine the power consumption of the non-volatile memory. The program process is achieved by trapping electrons in the gate oxide layer to change the device turn-on voltage. In practical applications, devices work in various environments so that electrons may obtain energy from external stressing, such as thermal energy, causing random tunneling from gate oxygen to the channel when the electron energy is large enough to cross the barrier height. Therefore, as the final step of device performance characterization, we have studied the data retention capability of the memory, which is directly reflected by changing the waiting time. The traditional method of evaluating memory data retention is from the shift of threshold voltage of the device, determined by the transfer characteristics at different intervals. One disadvantage of this method is that it may cause the device written when sweeping the transfer characteristic curve, thereby causing inaccuracy of the data. For the charge retention test, we adopted a simple and accurate method, which ensures the accuracy of the test results. After performing a program operation $(+6 \mathrm{v}$ pulse during $1 \mathrm{~ms}$ ), the 1-state current of the memory is measured only by applying a drain voltage of $100 \mathrm{mV}$ at different waiting times. As shown in Figure 3f, the 1-state current decays exponentially with the increasing wait time, fitted well with $I_{D S}=I_{0}+A \times e^{-\frac{t}{\tau}}$ [23]. Nothing but, when the wait time is 100 , the 1-state current only shows a slight drop, which can still guarantee the accuracy of the stored information. Compared with the poor data storage capacity of volatile memory (approximately on the millisecond timescale) [29], the refresh time is increased by about $10^{5}$ times, which will greatly reduce the power consumption of the memory. 

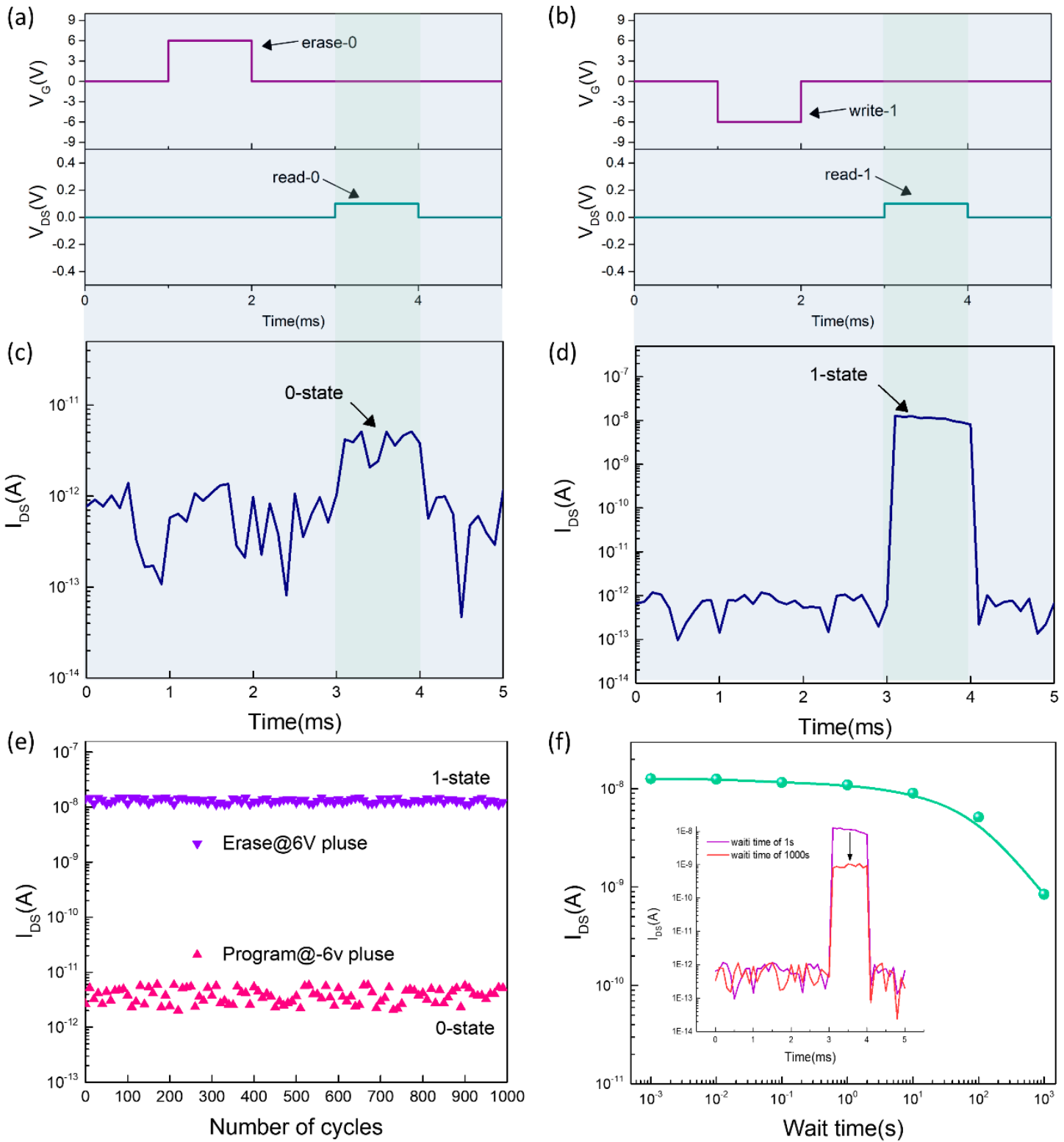

Figure 3. Demonstration of the write/erase operation. $(\mathbf{a}, \mathbf{c})$ The erase operation with $+6 \mathrm{v}$ pulse signal and read 0 -state, respectively. (b,d) The program operation with $-6 \mathrm{v}$ pulse signal and read 1-state, respectively. (e) The endurance testing of memory cell in 1000 cycles, and no performance reduction is observed. (f) The exponentially decay characteristic with wait time increasing. The refresh time of $100 \mathrm{~s}$ is obtained from the decay curve. The inset of (f) shows the output signal degradation when the wait times increase from 1 to $100 \mathrm{~s}$.

It is well known that the interface state charge may cause the hysteresis effect, which induces the threshold voltage variation. The hysteresis effect induced by the interface trap charge is always uncontrollable. In order to better illustrate that the device performance obtained is not accidental, we conducted a device repeatability test, as shown in Figure 4 . The six devices we selected are distributed in different areas of the same sample. Memory windows sweeping is performed at different sweep voltages for all devices. From Figure 4, these six devices show good repeatability, although there is a little fluctuation in the transfer characteristic, the influence of these fluctuations on the memory cell is negligible. Finally, we compared the device performance with other literatures, exhibited in Table 1. It can be found that the device we proposed not only can obtain a comparable device performance 
with the other memory, but also greatly simplifies the manufacturing process, which provides the possibility for large-scale manufacturing.
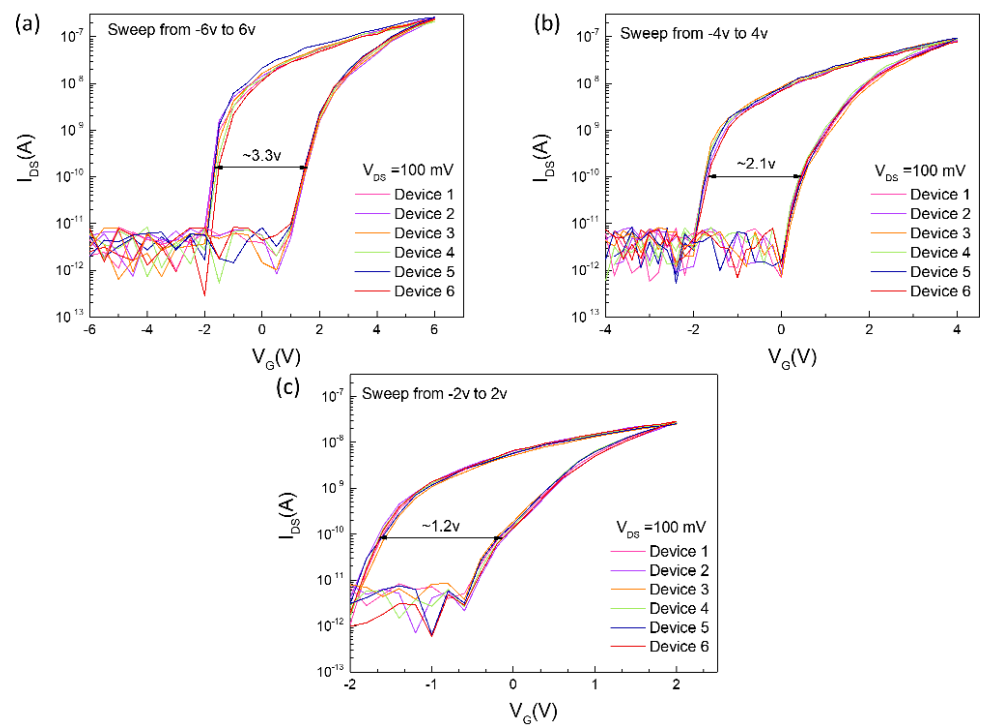

Figure 4. Device-to-device repeatability test from six different regions. (a) Sweeping in $\pm 6 \mathrm{v}$. (b) Sweeping in $\pm 4 \mathrm{v}$. (c) Sweeping in $\pm 6 \mathrm{v}$.

Table 1. Comparison of the performance of memory with other literatures.

\begin{tabular}{|c|c|c|c|c|c|c|c|}
\hline Ref. & Materials & $\begin{array}{l}\text { Program/Erase } \\
\text { Ratio }\end{array}$ & $\begin{array}{l}\text { Program/Erase } \\
\text { Voltage }\end{array}$ & $\begin{array}{l}\text { Write } \\
\text { Speed }\end{array}$ & $\begin{array}{c}\text { Date Retention } \\
\text { Time }\end{array}$ & $\begin{array}{l}\text { Process } \\
\text { Complexity }\end{array}$ & $\begin{array}{l}\text { Large-Scale } \\
\text { Preparation }\end{array}$ \\
\hline Our result & $\mathrm{MoS}_{2} / \mathrm{LaAlO}_{3}$ & $\sim 1 \times 10^{3}$ & $\pm 6 \mathrm{v}$ & $1 \mathrm{~ms}$ & $1000 \mathrm{~s}$ & I & $\sqrt{ }$ \\
\hline [22] & $\mathrm{HfO}_{2} / \mathrm{Graphene} / \mathrm{HfO}_{2} / \mathrm{MoS}_{2}$ & $\sim 1 \times 10^{3}$ & $\pm 18 \mathrm{v}$ & $100 \mathrm{~ms}$ & 10 year & II & $x$ \\
\hline [27] & $\mathrm{Al}_{2} \mathrm{O}_{3} / \mathrm{HfO}_{2} / \mathrm{Al}_{2} \mathrm{O}_{3} / \mathrm{MoS}_{2}$ & $<1 \times 10^{3}$ & $\pm 26 \mathrm{v}$ & $200 \mathrm{~ms}$ & - & II & $x$ \\
\hline [29] & $\mathrm{MoS}_{2} / \mathrm{BN} / \mathrm{WSe}{ }_{2}$ & $\sim 1 \times 10^{2}$ & $\pm 5 \mathrm{v}$ & $40 \mathrm{~ns}$ & $14 \mathrm{~s}$ & III & $x$ \\
\hline [30] & $\mathrm{HfO}_{2} / \mathrm{Metal} / \mathrm{HfO}_{2} / \mathrm{MoS}_{2}$ & $\sim 1 \times 10^{5}$ & $\pm 15 \mathrm{v}$ & $100 \mathrm{~ms}$ & 10 year & II & $\times$ \\
\hline
\end{tabular}

Process complexity progressively increases from I to IV.

\section{Mechanism Analysis}

The current main charge storage devices are based on floating gate structures [22,23,30,31], as shown in Figure S5. During the programming process, the electrons tunnel pass through the barrier layer and are trapped by the floating gate, whereas electrons are de-trapped during the erase operation. The trapping layer plays a leading role for charge storage. Due to the better compactness and higher crystallinity of binary oxides, there are only a few trap states in binary oxides. Therefore, the contribution of the charge restrained by the trap state to the operating window is insignificant. However, for the memory device we proposed, there is not the capture layer. Lanthanum incorporation in $\mathrm{Al}_{2} \mathrm{O}_{3}$ broke the original lattice structure of $\mathrm{Al}_{2} \mathrm{O}_{3}$, causing a mass of electron traps to exist in $\mathrm{LaAlO}_{3}$, as shown in Figure 5b. The green hollow circle represents the electron trap in oxide. During the programming process, the electron trap is filled with electrons, and offset with the fixed charge of the oxide layer (blue solid circles). This electron transfer process causes the threshold voltage to drift.

The hysteresis effect in the $\mathrm{MoS}_{2}$ transistor based on $\mathrm{SiO}_{2}$ may be dominant by the interface trap charge, because it is seriously affected by environmental factors [31,32]. That will not have good device-to-device reproducibility, whereas the devices we proposed exhibit good repeatability, as shown in Figure 4. Moreover, in some literatures, $\mathrm{Al}_{2} \mathrm{O}_{3}$ encapsulation is used to reduce the hysteresis effect [33]. Although the hysteresis effect in the $\mathrm{MoS}_{2}$ transistor based on $\mathrm{SiO}_{2}$ has a false effect that is similar to a memory device, there are many shortcomings in $\mathrm{SiO}_{2}$. As shown in Figure S6, despite having a large memory windows, the program/erase ratio is still less than $1 \times 10^{2}$ due to the small dielectric constant of $\mathrm{SiO}_{2}$. Not only that, the program/erase voltage has reached \pm 40 , which is 
disadvantageous for the endurance of memories. However, our proposed memory cell only needs a \pm 6 operation voltage to achieve a program/erase ratio of $1 \times 10^{3}$, which shows great advantages in storage applications.

(a)

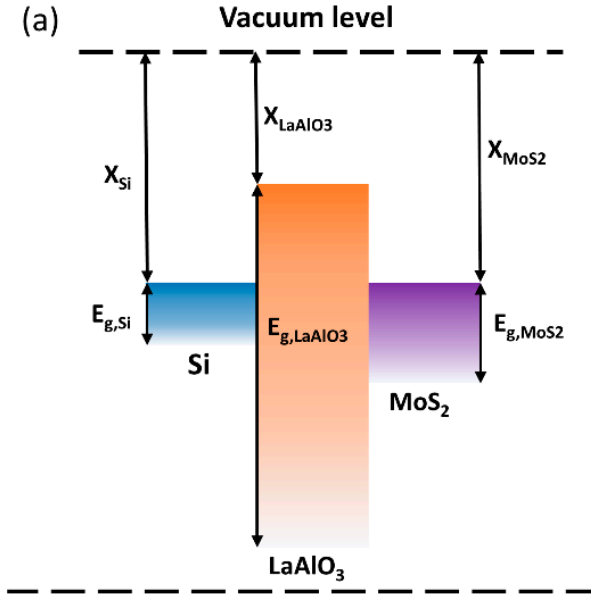

(b)

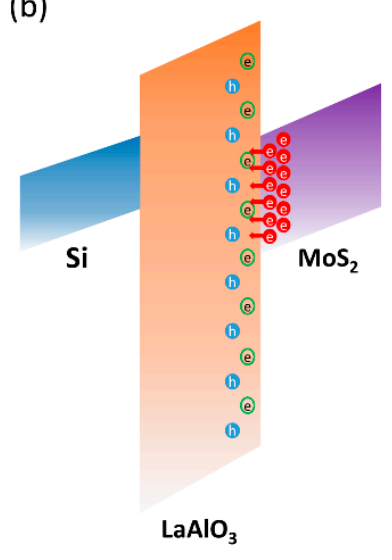

(c)

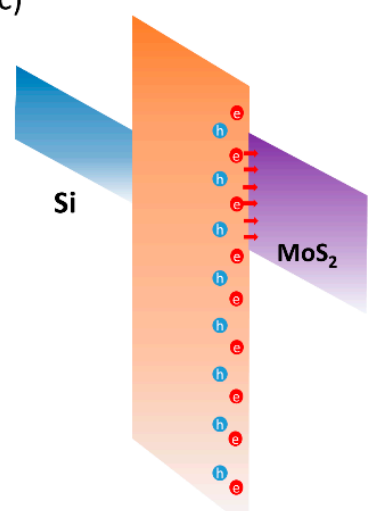

$\mathrm{LaAlO}_{3}$ (d)

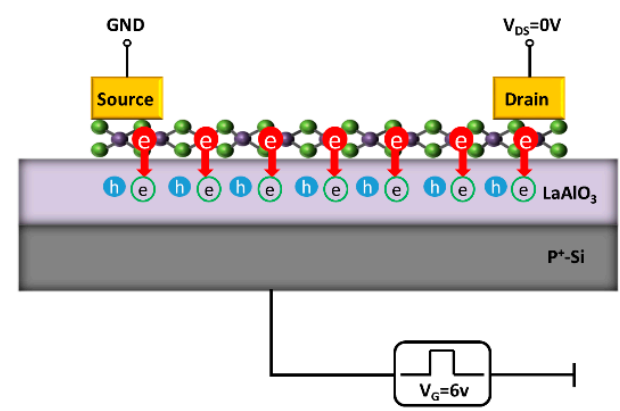

(e)

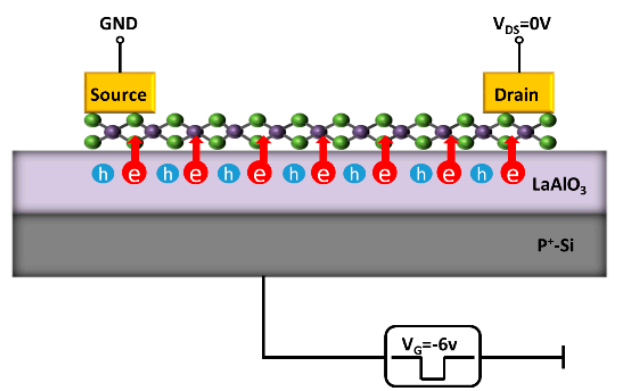

Figure 5. (a) The energy band diagram without the applied voltage $\left(\mathrm{X}_{\mathrm{Si}} \approx 4.13 \mathrm{eV}, \mathrm{E}_{\mathrm{g}, \mathrm{Si}} \approx 4.13 \mathrm{eV}\right.$, $\left.\mathrm{X}_{\mathrm{LaAlO} 3} \approx 2.39 \mathrm{eV}, \mathrm{E}_{\mathrm{g}, \mathrm{LaAlO} 3} \approx 6.5 \mathrm{eV}, \mathrm{X}_{\mathrm{MoS2}} \approx 4.2 \mathrm{eV}, \mathrm{E}_{\mathrm{g}, \mathrm{MoS} 2} \approx 1.8 \mathrm{eV}\right)$. (b,d) The transfer of electrons in the program operation. $(\mathbf{c}, \mathbf{e})$ The transfer of electrons in the erase operation.

\section{Conclusions}

In this work, we have manufactured a charge trapped non-volatile memory based on $\mathrm{MoS}_{2}$ transistors with extremely strong information storage capabilities. This memory cell exhibits a large memory windows of $3.3 \mathrm{v}$ (sweeping from -6 to $6 \mathrm{v}$ and back to $-6 \mathrm{v}$ ), ultra-fast writing speed (up to $1 \mathrm{~ms}$ ), stable program/erase ratio of $10^{3}$, and longer refresh time (approximately $100 \mathrm{~s}$ ) that avoids unnecessary power dissipation due to frequent refresh operations. At the same time, the simple back-gate process makes it well compatible with existing memory fabrication processes. All these impressive performances indicate that the charge trapping non-volatile memory based on $\mathrm{MoS}_{2}$ transistors will promote the development of a two-dimensional memory technology.

Supplementary Materials: The following are available online at http://www.mdpi.com/2079-4991/10/8/1471/s1, Figure S1: LaAlO3 film interface atomic force microscopy (AFM) topography, Figure S2: XPS spectrum of LaAlO3 film, Figure S3: O1s XPS spectrum of LaAlO3 film, Figure S4: Cross-sectional HRTEM images of sample, Figure S5: Non-volatile Memory Cells Based on MoS2/Graphene Heterostructures, Figure S6: MoS2/SiO2 back gate transistor.

Author Contributions: Writing—original draft preparation, K.Y.; writing—review and editing, K.Y.; visualization, W.Y.; resources, T.H.; supervision, S.W.; funding acquisition, H.L. All authors have read and agreed to the published version of the manuscript.

Funding: This research was funded by the National Natural Science Foundation of China (grant no. U1866212), the Laboratory Open Fund of Beijing Smart-chip Microelectronics Technology Co., Ltd. (grant no. SGITZXDDKJQT2002303), and the Innovation Foundation of Radiation Application (grant no. KFZC2018040206).

Conflicts of Interest: The authors declare no conflict of interest. 


\section{References}

1. International Technology Roadmap for Semiconductors 2.0 (ITRS, 2015). Available online: https://www. semiconductors.org/resources/2015-international-technology-roadmap-for-semiconductors-itrs/ (accessed on 8 June 2020).

2. Britnell, L.; Gorbachev, R.V.; Jalil, R.; Belle, B.D.; Schedin, F.; Katsnelson, M.I.; Eaves, L.; Morozov, S.V.; Mayorov, A.S.; Peres, N.M.R.; et al. Electron tunneling through ultrathin boron nitride crystalline barriers. Nano Lett. 2012, 12, 1707-1710. [CrossRef]

3. Liu, L.; Han, H.; Xu, L.; Zhou, J.; Zhao, C.; Ding, S.; Shi, H.; Xiao, M.; Ding, L.; Jin, C.; et al. Aligned, high-density semiconducting carbon nanotube arrays for high-performance electronics. Science 2020, 368, 850-856. [CrossRef] [PubMed]

4. Li, S.; Tian, M.; Gu, C.; Wang, R.; Wang, M.; Xiong, X.; Li, X.; Huang, R.; Wu, Y. BEOL Compatible 15-nm Channel Length Ultrathin Indium-Tin-Oxide Transistors with Ion $=970 \mu \mathrm{A} / \mu \mathrm{m}$ and On/off Ratio Near 1011 at Vds $=0.5$ V. In Proceedings of the 2019 IEEE International Electron Devices Meeting (IEDM), San Francisco, CA, USA, 7-11 December 2019.

5. Lembke, D.; Bertolazzi, S.; Kis, A. Single-layer MoS2 electronics. Acc. Chem. Res. 2015, 48, 100-110. [CrossRef] [PubMed]

6. He, Q.; He, Q.; Zeng, Z.; Yin, Z.; Li, H.; Wu, S.; Huang, X.; Zhang, H. Fabrication of flexible MoS2 thin-film transistor arrays for practical gas-sensing applications. Small 2012, 8, 2994-2999. [CrossRef]

7. Britnell, L.; Ribeiro, R.M.; Eckmann, A.; Jalil, R.; Belle, B.D.; Mishchenko, A.; Kim, Y.-J.; Gorbachev, R.V.; Georgiou, T.; Morozov, S.V.; et al. Strong Light-Matter Interactions in Heterostructures of Atomically Thin Films. Science 2013, 340, 1311-1314. [CrossRef]

8. Yu, W.J.; Liu, Y.; Zhou, H.; Yin, A.; Li, Z.; Huang, Y.; Duan, X. Highly efficient gate-tunable photocurrent generation in vertical heterostructures of layered materials. Nat. Nanotechnol. 2013, 8, 952-958. [CrossRef] [PubMed]

9. Shi, Y.; Liang, X.; Yuan, B.; Chen, V.; Li, H.; Hui, F.; Yu, Z.; Yuan, F.; Pop, E.; Wong, H.-S.P.; et al. Electronic synapses made of layered two-dimensional materials. Nat. Electron. 2018, 1, 458-465. [CrossRef]

10. Wang, M.; Cai, S.; Pan, C.; Wang, C.; Lian, X.; Zhuo, Y.; Xu, K.; Cao, T.; Pan, X.; Wang, B.; et al. Robust memristors based on layered two-dimensional materials. Nat. Electron. 2018, 1, 130-136. [CrossRef]

11. Sangwan, V.K.; Lee, H.; Bergeron, H.; Balla, I.; Beck, M.E.; Chen, K.-S.; Hersam, M.C. Multi-terminal memtransistors from polycrystalline monolayer molybdenum disulfide. Nature 2018, 554, 500-504. [CrossRef]

12. Smithe, K.K.H.; Suryavanshi, S.V.; Munoz Rojo, M.; Tedjarati, A.D.; Pop, E. Low Variability in Synthetic Monolayer MoS2 Devices. ACS Nano 2017, 11, 8456-8463. [CrossRef]

13. Li, W.; Zhou, J.; Cai, S.; Yu, Z.; Zhang, J.; Fang, N.; Li, T.; Wu, Y.; Chen, T.; Xie, X.; et al. Uniform and ultrathin high-K gate dielectrics for two-dimensional electronic devices. Nat. Electron. 2019, 2, 563-571. [CrossRef]

14. Si, M.; Su, C.-J.; Jiang, C.; Conrad, N.J.; Zhou, H.; Maize, K.D.; Qiu, G.; Wu, C.-T.; Shakouri, A.; Alam, M.A.; et al. Steep-slope hysteresis-free negative capacitance MoS2 transistors. Nat. Nanotechnol. 2018, 13, $24-28$. [CrossRef]

15. Wachter, S.; Polyushkin, D.K.; Bethge, O.; Mueller, T. A microprocessor based on a two-dimensional semiconductor. Nat. Commun. 2017, 8, 14948. [CrossRef] [PubMed]

16. Yu, W.J.; Li, Z.; Zhou, H.; Chen, Y.; Wang, Y.; Huang, Y.; Duan, X. Vertically stacked multi-heterostructures of layered materials for logic transistors and complementary inverters. Nat. Mater. 2013, 12, $246-252$. [CrossRef] [PubMed]

17. Choi, M.S.; Lee, G.H.; Yu, Y.-J.; Lee, D.-Y. Controlled charge trapping by molybdenum disulphide and graphene in ultrathin heterostructured memory devices. Nat. Commun. 2013, 4, 1624. [CrossRef] [PubMed]

18. Roy, K.; Padmanabhan, M.; Goswami, S.; Sai, T.P.; Ramalingam, G.; Raghavan, S. Graphene-MoS2 hybrid structures for multifunctional photoresponsive memory devices. Nat. Nanotechnol. 2013, 8, 826-830. [CrossRef] [PubMed]

19. Kappera, R.; Voiry, D.; Yalcin, S.E.; Branch, B.; Gupta, G.; Mohite, A.D.; Chhowalla, M. Phase-engineered low-resistance contacts for ultrathin MoS2 transistors. Nat. Mater. 2014, 13, 1128-1134. [CrossRef] [PubMed]

20. Yang, J.J.; Strukov, D.B.; Stewart, D.R. Memristive devices for computing. Nat. Nanotechnol. 2013, 8, 13-24. [CrossRef] 
21. JEDEC Solid State Technology Association Standard JESD79-2b 65 (2005). Available online: http://cs.ecs. baylor.edu/ \{\}maurer/CSI5338/JESD79-2B.pdf (accessed on 8 June 2020).

22. Bertolazzi, S.; Krasnozhon, D.; Kis, A. Nonvolatile Memory Cells Based on MoS2/Graphene Heterostructures. ACS Nano 2013, 7, 3246-3252. [CrossRef]

23. Liu, C.; Yan, X.; Song, X.; Ding, S.; Zhang, D.W.; Zhou, P. A semi-floating gate memory based on van der Waals heterostructures for quasi-non-volatile applications. Nat. Nanotechnol. 2018, 13, 404-410. [CrossRef]

24. Zade, D.; Sato, S.; Kakushima, K.; Srivastava, A.; Ahmet, P.; Tsutsui, K.; Nishiyama, A.; Sugii, N.; Natori, K.; Hattori, T.; et al. Effects of La2O3 incorporation in $\mathrm{HfO} 2$ gated nMOSFETs on low-frequency noise. Microelectron. Reliab. 2011, 51, 746-750. [CrossRef]

25. Umezawa, N.; Shiraishi, K.; Sugino, S.; Tachibana, A.; Ohmori, K.; Kakushima, K.; Iwai, H.; Chikyow, T.; Ohno, T.; Nara, Y.; et al. Suppression of oxygen vacancy formation in Hf-based high-k dielectrics by lanthanum incorporation. Appl. Phys. Lett. 2007, 91. [CrossRef]

26. Nan, H.; Wang, Z.; Wang, W.; Liang, Z.; Lu, Y.; Chen, Q.; He, D.; Tan, P.; Miao, F.; Wang, X.; et al. Strong Photoluminescence Enhancement of MoS2 through Defect Engineering and Oxygen Bonding. ACS Nano 2014, 8, 5738-5745. [CrossRef] [PubMed]

27. Zhang, E.; Wang, W.; Zhang, C.; Jin, Y.; Zhu, G.; Sun, Q.; Zhang, D.W.; Zhou, P.; Xiu, F. Tunable Charge-Trap Memory Based on Few-Layer MoS2. ACS Nano 2015, 9, 612-619. [CrossRef] [PubMed]

28. Guo, Y.; Wei, X.; Shu, J.; Liu, B.; Yin, J.; Guan, C.; Han, Y.; Gao, S.; Chen, Q. Charge trapping at the MoS2-SiO2 interface and its effects on the characteristics of MoS2 metal-oxide-semiconductor field effect transistors. Appl. Phys. Lett. 2015, 106. [CrossRef]

29. Li, J.; Liu, L.; Chen, X.; Liu, C.; Wang, J.; Hu, W.; Zhang, D.W.; Zhou, P. Symmetric Ultrafast Writing and Erasing Speeds in Quasi-Nonvolatile Memory via van der Waals Heterostructures. Adv. Mater. 2019, 31, e1808035. [CrossRef]

30. Wang, J.; Zou, X.; Xiao, X.; Xu, L.; Wang, C.; Jiang, C.; Ho, J.C.; Wang, T.; Li, J.; Liao, L. Floating gate memory-based monolayer MoS2 transistor with metal nanocrystals embedded in the gate dielectrics. Small 2015, 11, 208-213. [CrossRef] [PubMed]

31. Di Bartolomeo, A.; Genovese, L.; Giubileo, F.; Iemmo, L.; Luongo, G.; Foller, T.; Schleberger, M. Hysteresis in the transfer characteristics of MoS2 transistors. 2D Mater. 2017, 5. [CrossRef]

32. Urban, F.; Giubileo, F.; Grillo, A.; Iemmo, L.; Luongo, G.; Passacantando, M.; Foller, T.; Madauß, L.; Pollmann, E.; Geller, M.P.; et al. Gas dependent hysteresis in MoS2 field effect transistors. 2D Mater. 2016, 6. [CrossRef]

33. Illarionov, Y.Y.; Smithe, K.K.H.; Waltl, M.; Knobloch, T.; Pop, E.; Grasser, T. Improved Hysteresis and Reliability of MoS2 Transistors With High-Quality CVD Growth and Al2O3 Encapsulation. IEEE Electron. Device Lett. 2017, 38, 1763-1766. [CrossRef]

(C) 2020 by the authors. Licensee MDPI, Basel, Switzerland. This article is an open access article distributed under the terms and conditions of the Creative Commons Attribution (CC BY) license (http://creativecommons.org/licenses/by/4.0/). 